\title{
Relapse after conservative surgery combined with triptorelin acetate versus conservative surgery only in women with focal adenomyosis: study protocol for a multicenter, prospective, randomized controlled trial
}

\author{
Wenwen Wang ${ }^{1}$, Xiangyi Ma ${ }^{1}$, Wei Zhang ${ }^{2}$, Zhiying $\mathrm{Li}^{3}$, Yan Wang ${ }^{4}$, Zhiying Yu ${ }^{5}$, Chunlian Zhang ${ }^{6}$, Li Hong ${ }^{7}$, \\ Ruoyu Luo $^{7}$, Hui Xing ${ }^{8}$, Wuliang Wang ${ }^{9}$, Qingfen Yue ${ }^{10}$, Jia Wei ${ }^{1}$, Minli Zhang ${ }^{1}$ and Shixuan Wang ${ }^{1 *}$
}

\begin{abstract}
Background: The preservation of fertility and integrity of the reproductive organs has increasingly been of concern to most women with adenomyosis. Adenomyomectomy is conservative surgery that is now widely applied; however, relapse is a serious problem after the operation. Postoperative treatment, such as gonadotropin-releasing hormone agonist ( $\mathrm{GnRHa}$ ) has been suggested to result in reducing the rate of disease recurrence. However, there is still a lack of evidence from randomized clinical trials examining the efficacy of GnRHa in decreasing the postoperative recurrence rate.
\end{abstract}

Method/design: Relapse after conservative surgery combined with triptorelin acetate versus conservative surgery only in women with focal adenomyosis is a multicenter, prospective, randomized controlled trial. The primary outcome is relapse assessed using a visual analogue scale (VRS) and numeric rating scale (NRS), pictorial blood loss assessment chart (PBAC) score, and the size of the uterus and the lesion as measured by two/three-dimensional color doppler ultrasonography (2D/3D-CDUS) or magnetic resonance imaging (MRI). The secondary outcomes include quality of life, clinical pregnancy, ovarian reserve, adverse events, assessment by the Short Form (36) Health Survey and Female Sexual Function index, serum follicle-stimulating hormone, estradiol levels, and anti-Muellerian hormone and so on. All these indexes are measured at 3, 6, 12, 18, 24, 30, and 36 months after conservative surgery.

Discussion: The result of this large, multicenter randomized trial will provide evidence for one of the strategies of long-term management in focal adenomyosis after conservative operation.

Trial registration: Chinese Clinical Trial Registry: ChiCTR1800014340. Registered on 6 January 2018.

Keywords: Focal adenomyosis, Adenomyomectomy, Relapse, Triptorelin acetate

\footnotetext{
* Correspondence: shixuanwang@tjh.tjmu.edu.cn

'Department of Obstetrics and Gynecology, Tongji Hospital, Tongji Medical

College, Huazhong University of Science and Technology, Wuhan, Hubei,

China

Full list of author information is available at the end of the article
}

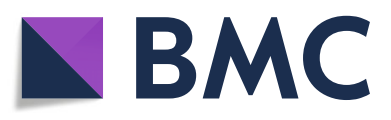

(- The Author(s). 2020 Open Access This article is licensed under a Creative Commons Attribution 4.0 International License, which permits use, sharing, adaptation, distribution and reproduction in any medium or format, as long as you give appropriate credit to the original author(s) and the source, provide a link to the Creative Commons licence, and indicate if changes were made. The images or other third party material in this article are included in the article's Creative Commons licence, unless indicated otherwise in a credit line to the material. If material is not included in the article's Creative Commons licence and your intended use is not permitted by statutory regulation or exceeds the permitted use, you will need to obtain permission directly from the copyright holder. To view a copy of this licence, visit http://creativecommons.org/licenses/by/4.0/. The Creative Commons Public Domain Dedication waiver (http://creativecommons.org/publicdomain/zero/1.0/) applies to the data made available in this article, unless otherwise stated in a credit line to the data. 


\section{Background}

Adenomyosis is a common gynecologic benign disorder characterized by aberrant presence of endometrial glands and stromal cells within the myometrium [1-3]. The main symptoms are dysmenorrhea, chronic pelvic pain, dyspareunia, abnormal uterine bleeding (especially with heavy menstrual bleeding) and infertility, which seriously affect quality of life and work productivity in women of reproductive age $[4,5]$.

Hysterectomy is considered as radical therapy for patients who are refractory to other treatments or who do not wish to preserve their fertility $[6,7]$. However, many patients of reproductive age are very concerned to preserve their fertility and reproductive organs [8]. Medical treatments or conservative surgery are options for these patients. The former include non-steroidal anti-inflammatory drugs or hormone regulating drugs, which can maintain a hypoestrogenic state, such as gonadotropin releasing hormone agonist (GnRHa), danazol, progestogens, or oral contraceptive pills $[9,10]$. GnRHa has been the best acceptable treatment for patients during the past 20 years [11]. Unfortunately, these medicines may only provisionally improve symptoms and often lead to serious side effects [12]. Therefore, conservative surgery is an alternative; depending on the extent of the disease, the surgical options are adenomyomectomy (for localized adenomyosis) and partial adenomyomectomy (for diffuse adenomyosis) [4, 13]. The rate of pain relief after conservative surgery is higher in patients with focal adenomyosis than in those with diffuse adenomyosis [14]. However, discrepancy of relapse rate was the main issue for this treatment, due to the residual ratio of lesions. The cause was put down to the unclear cut line between focus and normal myometrial tissue [14]. How to control recurrence after surgery has become the current priority.

Recently, some prospective or retrospective studies $[7,15,16]$ have confirmed that surgical-medical treatment is a more effective option for the treatment of symptomatic relief in focal adenomyosis than surgical treatment alone. However, there is still a lack of evidence from randomized controlled studies to confirm whether GnRH agonists could decrease the disease recurrence rate after conservative surgery in women with focal adenomyosis. This study is a multicenter, randomized, parallel controlled trial comparing the efficacy of triptorelin acetate in protecting against postoperative relapse in patients with focal adenomyosis.

\section{Method/design}

Participants $(n=308)$ who have undergone complete excision of focal adenomyosis will be randomly assigned 1: 1 to the treatment group (triptorelin acetate) or the control group. Patients will be recruited at 10 hospitals across mid-west China. All research units have been approved by the ethics committees. Every patient will sign an informed consent form prior to this study. This report follows the Standard Protocol Items: Recommendations for Interventional Trials (SPIRIT) guideline (Additional file 1).

\section{Inclusion criteria}

The inclusion criteria are as follows:

1. Women aged $\geq 18$ and $\leq 45$ years

2. Women with pathologically confirmed diagnosis of focal adenomyosis

3. Women who accept complete excision of adenomyosis and have not taken any steroid hormone therapy in the 3 months before surgery

4. Women who are healthy before and are not pregnant

5. Women who can comply with the study procedures and give written informed consent

\section{Exclusion criteria}

1. Women who are also participating in other clinical trials at the same time

2. Women who have concomitantly been diagnosed with ovarian endometrioma, deep infiltrating endometriosis, or multiple leiomyomas

3. Women with congenital uterine abnormalities such as uterine malformation (unicornis uterus, septate uterus, or duplex uterus) or acute genital inflammation or malignant tumor

4. Women who are pregnant

5. Women who have taken steroid hormone therapy in the 3 months before surgery

6. Women with hereditary disease, blood disease, liver or kidney dysfunction, or malnutrition disorders that cause anemia

7. Women, who may get contraindications, cannot tolerate surgery or are allergic to triptorelin acetate

8. Women who are unable to comply with the study procedures and give written informed consent

\section{Screening and enrollment}

Previous medical history and current medication status are reviewed in the standardized case report forms. A physical examination and imaging such as two/three-dimensional color doppler ultrasonography (2D/3D-CDUS) or magnetic resonance imaging (MRI) are performed. Laboratory measurements include measurement of serum follicle-stimulating hormone (FSH), estradiol (E2), antiMuellerian hormone (AMH), cancer antigen 125 (CA125), and safety assays including routine analysis of blood, urine, liver function, renal function, hepatitis virus, HIV, syphilis, and coagulation; electrocardiogram (ECG) and radiography are performed in the local departments at the 
study sites. Either laparoscopy or transabdominal surgery is performed to completely remove all clinically recognizable lesions in patients who are suspected to have focal adenomyosis.

Written informed consent will be obtained from the patients after surgery. Quality of life, degree of pain, and menstrual volume will be recorded using the Short Form (36) Health Survey (SF-36), Female Sexual Function Index (FSFI), pictorial blood loss assessment chart (PBAC) score, visual analogue scale (VAS) and numeric rating scale (NRS) after confirming by histologic assessment. A schedule of enrollment, interventions, and assessment is provided Table 1.

\section{Adenomyomectomy}

Laparotomy, hysteroscopy, or laparoscopy are performed in this study. During the laparoscopy, patients are in the lithotomy position under general anesthesia with endotracheal intubation. After sterilization, a pneumoperitoneum is created using carbon dioxide gas at $13 \mathrm{mmHg}$ (Karl Storz GmbH \& Co. KG, Tuttlingen, Germany). Surgery is performed using four surgical trocars. The supine position and a median incision are suitable for laparotomy. Hysteroscopic surgery is conducted for type 0 or type 1 adenomyoma (Karl Storz GmbH \& Co. KG, Tuttlingen, Germany). The surgical approach is adenomyomectomy. The principles of these surgical options include complete removal of clinically visible lesions, maintaining the integrity of the uterine wall, and retaining the integrity of the uterine cavity as far as possible. The duration of surgery, size of the focus, blood loss, and the integrity of the uterine cavity should be recorded.

\section{Interventions}

Eligible participants are assigned by simple randomization in a 1:1 ratio to a group receiving surgery only (group A) or to a group receiving surgery plus $\mathrm{GnRH}$ agonists (group B). The GnRH agonist is administered in the intervention group as a $3.75 \mathrm{mg}$ intramuscular injection (Diphereline, Ipsen, France) on the first day of menstruation after surgery, then once every 4 weeks for six courses. The signs and symptoms of hypoestrogenism, including hot flushes, night sweats, sleep disorders, abnormal emotions and osteoporosis, could be caused by the GnRH agonist; these should be carefully evaluated and participants could be treated with tibolone $1.25 \mathrm{mg}$ per day as add-back therapy to maintain serum estradiol at $30-50 \mathrm{pg} / \mathrm{ml}$ [17].

The intervention will be discontinued or modified if an intolerable adverse reaction occurs in group B. Color doppler ultrasonography and a steroid hormone test on the 3rd and 6th month after conservative surgery can help to monitor adherence. During the trial, other medications, such as steroid hormone or herbal medicine, or another intervention, such as levonorgestrel-releasing intrauterine system (LNG-IUS) will be prohibited.

\section{Randomization}

Eligible participants are assigned by simple randomization in a 1:1 ratio to one of two groups. The sequence of randomization has been set up by biostatisticians in the data coordination center using Microsoft Excel and the "RAND ()" function. The original sequence is safely kept by the staff in the data coordination center, and it has been put into the online central randomization system by these staff members, who are not involved in enrolling subjects. The sequence is not accessible to any

Table 1 Schedule of screening, enrollment and assessment

\begin{tabular}{|c|c|c|c|c|c|c|c|c|c|}
\hline \multirow[t]{2}{*}{ Evaluation } & \multirow[t]{2}{*}{ Screening } & \multirow[t]{2}{*}{ Enrollment } & \multicolumn{7}{|c|}{ Time of follow up after surgery (month) } \\
\hline & & & 3 & 6 & 12 & 18 & 24 & 30 & 36 \\
\hline Written consent & & $\sqrt{ }$ & & & & & & & \\
\hline Inclusion/exclusion criteria & & $\sqrt{ }$ & & & & & & & \\
\hline Medical history & $\sqrt{ }$ & & & & & & & & \\
\hline Physical exam & $\sqrt{ }$ & & & & & & & & \\
\hline Safety test (preoperative examination) & $\sqrt{ }$ & & & & & & & & \\
\hline 2D/3D-CDUS/MRI & $\sqrt{ }$ & & $\sqrt{ }$ & $\sqrt{ }$ & $\sqrt{ }$ & $\sqrt{ }$ & $\sqrt{ }$ & $\sqrt{ }$ & $\sqrt{ }$ \\
\hline $\mathrm{FSH}, \mathrm{E}_{2}, \mathrm{AMH}, \mathrm{CA} 125$ & $\sqrt{ }$ & & $\sqrt{ }$ & $\sqrt{ }$ & $\sqrt{ }$ & $\sqrt{ }$ & $\sqrt{ }$ & $\sqrt{ }$ & $\sqrt{ }$ \\
\hline Side effects & & & $\sqrt{ }$ & $\sqrt{ }$ & $\sqrt{ }$ & $\sqrt{ }$ & $\sqrt{ }$ & $\sqrt{ }$ & $\sqrt{ }$ \\
\hline VAS and NRS & & $\sqrt{ }$ & $\sqrt{ }$ & $\sqrt{ }$ & $\sqrt{ }$ & $\sqrt{ }$ & $\sqrt{ }$ & $\sqrt{ }$ & $\sqrt{ }$ \\
\hline SF-36 and FSFI & & $\sqrt{ }$ & $\sqrt{ }$ & $\sqrt{ }$ & $\sqrt{ }$ & $\sqrt{ }$ & $\sqrt{ }$ & $\sqrt{ }$ & $\sqrt{ }$ \\
\hline PBAC score & & $\sqrt{ }$ & $\sqrt{ }$ & $\sqrt{ }$ & $\sqrt{ }$ & $\sqrt{ }$ & $\sqrt{ }$ & $\sqrt{ }$ & $\sqrt{ }$ \\
\hline
\end{tabular}

2D/3D-CDUS/MRI two/three-dimensional color doppler ultrasonography/magnetic resonance imaging, $F S H$ follicle stimulating hormone, $E_{2}$ estradiol, $A M H$ antiMuellerian hormone, CA125 cancer antigen 125, VAS visual analogue scale, NRS numeric rating scale, SF-36 Short Form-36 Health Survey, FSFI Female Sexual Function index, PBAC pictorial blood loss assessment chart 
investigators or study coordinators. If a subject fulfills the enrollment criteria, the authorized study coordinator will obtain the assignment for her. After randomization, both subjects and investigators are informed about the assignments.

\section{Outcome and outcome assessments}

The primary outcome is relapse, which is defined as recurrence of dysmenorrhea or pelvic pain, or menorrhagia, or local recurrence compared to the first follow up after surgery as confirmed by 2D/3D-CDUS/MRI. Relapse as identified by 2D/3D-CDUS or MRI constitutes an increase $>1 \mathrm{~cm}$ in the maximal diameter of the suspected recrudescent focus during the follow-up period. The VRS and NRS are also applied for evaluation of dysmenorrhea or pelvic pain and the recurrence is defined as any increase in the scores and by participants asking for other medical treatments for relief of symptoms at the same time during follow up. The PBAC score is used to predict coagulation disorders in women with menorrhagia and recurrence is defined as a score $>100$. The proportion of participants with recurrence at $3,6,9,12,18,24,30$, and 36 months will be recorded.

The secondary outcomes include quality of life, clinically confirmed pregnancy, ovarian reserve, and adverse events (AE). Quality of life is assessed by the SF-36 and FSFI, as measures of health status and sexual functioning in women. Ovarian reserve is evaluated by measuring $\mathrm{FSH}, \mathrm{E}_{2}$, and $\mathrm{AMH}$; the first two are measured before surgery and during the follow-up period at the beginning of the menstrual cycle unless the participant is currently receiving treatment with triptorelin acetate. AMH can be measured at any time before and after surgery at the time of follow up. Differences in scores for eight dimensions (physical functioning, role-physical, bodily pain, general health, vitality, social functioning, role emotional and mental health) of the SF-36 and for the FSFI, and the mean values of $\mathrm{FSH}, \mathrm{E}_{2}$, and $\mathrm{AMH}$ for will be recorded for assessment of quality of life and ovarian reserve participants in each group of participants at 3, 6, 9,12, $18,24,30$, and 36 months. The participant's intention to become pregnant, the pregnancy rate, and outcome are recorded in a standard case report form, including details of abortion, premature delivery, and full-term delivery, to evaluate differences in pregnancy outcomes in each treatment arm at 36 months.

The follow-up procedure is acquired from the outpatient department records. Follow-up visits occur at 3, 6, $12,18,24,30$, and 36 months after conservative surgery.

Treatment-related AE are monitored at each visit. The $\mathrm{AE}$ are any unfavorable medical occurrences associated with the subject's participation in the research, whether or not considered related to the study intervention. Serious adverse events (SAE) are events that are temporally associated with the subject's participation in research that meet any of the following criteria: death, events that are life-threatening or severely or permanently disabling, events requiring in-patient hospitalization or prolongation of existing hospitalization, or any events deemed as serious by the local principal investigator. The proportion of patients with $\mathrm{AE}$ will be compared at 36 months to determine whether there is a difference between the groups.

\section{Data analysis \\ Sample size calculation}

Sample size was determined based on data from a prospective study demonstrating a relapse rate of about $49 \%$ in the group that received surgery alone and $28.1 \%$ in the group that received surgery and $\mathrm{GnRH}$ agonist treatment [7]. A two-tailed test with alpha set at 0.05 and $85 \%$ power is used to detect a minimum clinically meaningful difference between control and intervention groups. The minimal sample size is calculated as 139 participants in each group. In consideration of a dropout rate of $10 \%$, we will recruit 308 participants in total.

\section{Data collection}

Data are collected using a standard case report form. Data are de-identified before being entered into the database. The study site is regularly monitored and the database checked to ensure the accuracy of data collected. The data management, monitoring, and reporting of this study will comply with the International Conference on Harmonisation Good Clinical Practice ( $\mathrm{ICH}-$ $\mathrm{GCP})$ guidelines.

\section{Data analysis plan}

Data analysis and reporting will be conducted in accordance with the Consolidated Standards of Reporting Trials (CONSORT) 2010 Statement, as shown in our flow chart (Fig. 1), including the number of eligible participants and the number lost to follow up for various reasons. Data analysts will be blinded to the participants' assigned interventions. Unblinding of a participant's allocated intervention during the trial is permissible after blind verification and a submission of data locking proof by statistical analyst.

Intention to treat, which is based on the initial treatment intent, will be used as a foundation in our analysis. Participants who begin the allocated treatment are part of the trial, whether they finish it or not. The characteristics at baseline will be compared between the control and intervention groups. Continuous data will be summarized by the mean and standard deviation and the Wilcoxon rank sum test 


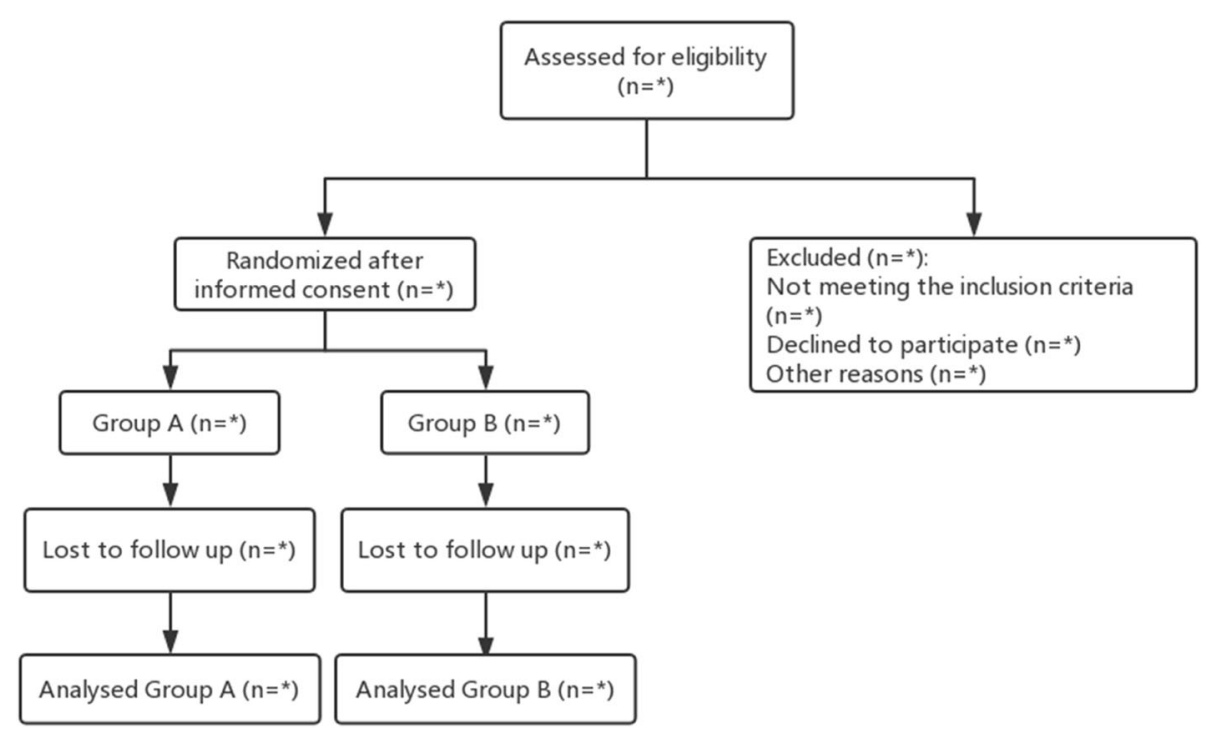

Fig. 1 Flowchart of this study

will be used to identify differences in baseline characteristic between the two groups. Categorical data will be described by number and percentage, using the Pearson chi-square test to compare data from the two groups.

The primary outcome measure is the difference in the recurrence rate between group A and group B after 3 years of follow up, which will be analyzed by the Pearson chi-square test. For efficacy parameters, such as the score for pelvic pain and dysmenorrhea, menstrual blood loss, and the size of the uterus and the lesion, will be analyzed using generalized estimating equations (GEE) or mixed effects model repeated measures (MMRM) to account for correlation among these observations at different follow-up points.

The variables from secondary outcomes, including scores on the SF-36 and the FSFI, the pregnancy rate, and pregnancy outcomes, are calculated during 36 months of follow up using GEE or MMRM analysis to compare groups A and B to identify differences between groups at different time points. The number of participants with adverse events (AE) or serious adverse events (SAE) will be calculated in each arm and taken into final statistical testing.

\section{Dissemination}

The results of the study will be published in a peerreviewed medical journal without using the services of a professional writer. Once agreed by the Steering Committee, the source data will be made available to share through national or international anonymized datasets.

\section{Discussion}

The preservation of fertility and the integrity of the reproductive organs is increasingly of concern to women with adenomyosis. Due to the disadvantage of medical treatments, adenomyomectomy was now increasingly applied. Remission from dysmenorrhea or dyspareunia after complete excision of the lesion has been reported as $50-94.7 \%$ and improvement in menorrhagia as $25-$ $80 \%$ [18]. Nevertheless, a symptomatic or local recurrence of the condition may occur. About $2.8-13.95 \%$ of patients relapsed at the end of first year in this study; $14.28-49 \%$ of patients had recurrence of disease in the following 24 months [18]. Therefore, the long-term management after surgery is the main issue to be addressed.

Recently, AI Jama retrospectively analyzed 18 patients who underwent adenomyomectomy and treatment with GnRHa for 24 weeks, 15 of whom had systematic improvement after 1 year of follow up [16]. Liu carried out a non-controlled descriptive study of 186 women with pathologically proven adenomyoma, who underwent ultramini-laparoscopic adenomyomectomy and a 6month course of goserelin acetate treatment. The rate of systematic recurrence was $9 \%$ after 3 years of follow up [19]. Wang et al. identified a significant decline in the relapse rate in the surgical-medical treatment group at the end of 2-year follow up in a prospective, nonrandomized study [7]. However, our retrospective analysis showed no difference between the control and intervention group at the end of the first or second year. It is noteworthy that there was a significant difference between the control and the GnRH agonist group in symptomatic relapse or relapse as identified on imaging 
studies at the end of the 36-months follow up. In other words, postoperative treatment might be applied to reduce the long-term recurrence rate. Whether or not we should use the postoperative method to prevent relapse in patients with adenomyosis is still unknown.

This is the first multicenter, prospective, randomized controlled trial, comparing the efficacy of GnRH agonist in reducing recurrence in patients with focal adenomyosis who have undergone adenomyomectomy. We cannot blind the trial participants and care providers to treatment allocation due to the drug-induced amenorrhea and/or series of side effects after GnRHa therapy, which will help patients and doctors identify the treatment. The lack of blinding might cause substantial bias, especially in the subjective judgment of patients, such pain scoring for the primary outcome. To solve this problem, we set up two types of pain scoring system to reduce the chance of subjective error judgment among patients. In addition, recurrence of dysmenorrhea is defined according to the patient's pain score and the need for changes in treatment, which can better evaluate progress after conservative treatment. We plan to enroll 308 participants from 10 teaching hospitals in China. The enrollment began in March 2018. At the time of manuscript preparation, more than 90 subjects have been enrolled. The result of this large multicenter randomized trial will provide level-I evidence for the strategy of long-term management of focal adenomyosis after conservative surgery.

\section{Trial status}

The protocol version is Version 1.0; March 8, 2018. Recruitment began on 8 March 2018. The expected date for recruitment completion is October-November 2019.

\section{Supplementary information}

Supplementary information accompanies this paper at https://doi.org/10. 1186/s13063-020-04294-2.

Additional file 1. SPIRIT checklist.

\section{Abbreviations}

2D/3D-CDUS: Two/three-dimensional color doppler ultrasonography; AMH: Anti-Muellerian hormone; E2: Estradiol; FSFI: Female Sexual Function index; FSH: follicle-stimulating hormone; GEE: Generalized estimating equations; GnRH: Gonadotropin-releasing hormone; MMRM: Mixed effects model repeated measures; MRI: Magnetic resonance imaging; NRS: Numeric rating scale; PBAC: Pictorial blood loss assessment chart; SF-36: Short Form (36) Health Survey; VAS: Visual analogue scale

\section{Acknowledgements}

We thank all subjects and physicians for volunteering their time to this study.

\section{Authors' contributions}

WW and XM contributed to the study design and manuscript drafting and revising; SW designed the study, is on the Steering Committee, and revised the manuscript; WZ, ZL, YW, ZY, CZ, LH, RL, HX, WW, QY, JW, and MZ were involved in the study concept and design and revision of the manuscript. All authors read and approved the final manuscript.

\section{Funding}

This is an investigator-initiated study without any grant from commercial funding agencies.

\section{Availability of data and materials}

No additional data available.

\section{Ethics approval and consent to participate}

Central ethical approval has been confirmed from the Tongji Hospital of Tongji Medical College of Huazhong University Medical Ethics Committee (reference approval number TJ-IRB20180310) and we will not begin recruiting at other centers in the trial until local ethical approval has been obtained. Written informed consent is obtained from the patients after screening.

\section{Consent for publication}

Not applicable.

\section{Competing interests}

The authors declare that they have no competing interests.

\section{Author details}

'Department of Obstetrics and Gynecology, Tongji Hospital, Tongji Medical College, Huazhong University of Science and Technology, Wuhan, Hubei, China. ${ }^{2}$ Department of Obstetrics and Gynecology, Zhongnan Hospital of Wuhan University, Wuhan University, Wuhan, Hubei, China. ${ }^{3}$ Department of Obstetrics and Gynecology, Affiliated Renhe Hospital, China Three Gorges University, Yichang, Hubei, China. ${ }^{4}$ Maternal and Child Hospital of Hubei Province, Tongji Medical College, Huazhong University of Science and Technology, Wuhan, Hubei, China. ${ }^{5}$ Department of Obstetrics and Gynecology, Shenzhen Second People's Hospital, Shenzhen, China.

${ }^{6}$ Department of Obstetrics and Gynecology, Affiliated Taihe Hospital of Hubei University of Medicine, Shiyan, Hubei, China. 'Department of Obstetrics and Gynecology, Renmin Hospital of Wuhan University Hubei General Hospital, Wuhan, Hubei, China. ${ }^{8}$ Department of Obstetrics and Gynecology, Xiangyang Central Hospital, Hubei University of Arts and Science, Xiangyang, Hubei, China. ${ }^{9}$ Department of Obstetrics and Gynecology, The Second Affiliated Hospital of Zhengzhou University, Zhengzhou, Henan, China. ${ }^{10}$ Department of Obstetrics and Gynecology, Luoyang Central Hospital Affiliated to Zhengzhou University, Luoyang, Henan, China.

Received: 12 June 2019 Accepted: 30 March 2020

Published online: 28 April 2020

References

1. Bergeron C, Amant F, Ferenczy A. Pathology and physiopathology of adenomyosis. Best Pract Res Clin Obstet Gynaecol. 2006;20(4):511-21. https://doi.org/10.1016/j.bpobgyn.2006.01.016.

2. Ferenczy A. Pathophysiology of adenomyosis. Hum Reprod Update. 1998: 4(4):312-22. https://doi.org/10.1093/humupd/4.4.312.

3. Levgur M. Diagnosis of adenomyosis: a review. J Reprod Med. 2007;52(3): 177-93.

4. Wood C. Surgical and medical treatment of adenomyosis. Hum Reprod Update. 1998;4(4):323-36. https://doi.org/10.1093/humupd/4.4.323.

5. Cheong Y, Cameron IT, Critchley HOD. Abnormal uterine bleeding. Br Med Bull. 2017;123(1):103-14. https://doi.org/10.1093/bmb/ldx027.

6. Osada H. Uterine adenomyosis and adenomyoma: the surgical approach. Fertil Steril. 2018;109(3):406-17. https://doi.org/10.1016/.ffertnstert.2018.01. 032.

7. Wang PH, Liu WM, Fuh JL, Cheng MH, Chao HT. Comparison of surgery alone and combined surgical-medical treatment in the management of symptomatic uterine adenomyoma. Fertil Steril. 2009;92(3):876-85. https:// doi.org/10.1016/j.fertnstert.2008.07.1744.

8. Streuli I, Dubuisson J, Santulli P, de Ziegler D, Batteux F, Chapron C. An update on the pharmacological management of adenomyosis. Expert Opin Pharmacother. 2014;15(16):2347-60. https://doi.org/10.1517/14656566.2014. 953055

9. Hirata JD, Moghissi KS, Ginsburg KA. Pregnancy after medical therapy of adenomyosis with a gonadotropin-releasing hormone agonist. Fertil Steril. 1993;59(2):444-5. 
10. Cheng MH, Wang PH. Uterine myoma: a condition amenable to medical therapy? Expert Opin Emerg Drugs. 2008;13(1):119-33. https://doi.org/10. 1517/14728214.13.1.119.

11. Tsui KH, Lee FK, Seow KM, Chang WC, Wang JW, Chen SU, et al. Conservative surgical treatment of adenomyosis to improve fertility: controversial values, indications, complications, and pregnancy outcomes. Taiwan J Obstet Gynecol. 2015;54(6):635-40. https://doi.org/10.1016/j.tjog. 2015.05.003.

12. Guo SW, Groothuis PG. Is it time for a paradigm shift in drug research and development in endometriosis/adenomyosis? Hum Reprod Update. 2018; 24(5):577-98. https://doi.org/10.1093/humupd/dmy020

13. Osada H, Silber S, Kakinuma T, Nagaishi M, Kato K, Kato O. Surgical procedure to conserve the uterus for future pregnancy in patients suffering from massive adenomyosis. Reprod BioMed Online. 2011;22(1):94-9. https:// doi.org/10.1016/.jbmo.2010.09.014

14. Grimbizis GF, Mikos T, Tarlatzis B. Uterus-sparing operative treatment for adenomyosis. Fertil Steril. 2014;101(2):472-87. https://doi.org/10.1016/j. fertnstert.2013.10.025.

15. Huang BS, Seow KM, Tsui KH, Huang CY, Lu YF, Wang PH. Fertility outcome of infertile women with adenomyosis treated with the combination of a conservative microsurgical technique and GnRH agonist: long-term followup in a series of nine patients. Taiwan J Obstet Gynecol. 2012;51(2):212-6. https://doi.org/10.1016/j.tjog.2012.04.008.

16. Al Jama FE. Management of adenomyosis in subfertile women and pregnancy outcome. Oman Med J. 2011;26(3):178-81. https://doi.org/10. 5001/omj.2011.43

17. Zupi E, Marconi D, Sbracia M, Zullo F, De Vivo B, Exacustos C, et al. Addback therapy in the treatment of endometriosis-associated pain. Fertil Steril. 2004;82(5):1303-8. https://doi.org/10.1016/j.fertnstert.2004.03.062.

18. Younes $\mathrm{G}$, Tulandi T. Conservative surgery for adenomyosis and results: a systematic review. J Minim Invasive Gynecol. 2017;25(2):265-76. https://doi. org/10.1016/j.jmig.2017.07.014,

19. Liu WM, Chen CH, Chiu LH, Tzeng CR. Long-term follow-up of severely symptomatic women with adenomyoma treated with combination therapy. Taiwan J Obstet Gynecol. 2013;52(1):85-9. https://doi.org/10.1016/j.tjog.2012. 12.002.

\section{Publisher's Note}

Springer Nature remains neutral with regard to jurisdictional claims in published maps and institutional affiliations.

Ready to submit your research? Choose BMC and benefit from:

- fast, convenient online submission

- thorough peer review by experienced researchers in your field

- rapid publication on acceptance

- support for research data, including large and complex data types

- gold Open Access which fosters wider collaboration and increased citations

- maximum visibility for your research: over $100 \mathrm{M}$ website views per year

At $\mathrm{BMC}$, research is always in progress.

Learn more biomedcentral.com/submissions 